\title{
Quantitative Analysis of Comprehensive Influence of Music Network Based on Logistic Regression and Bidirectional Clustering
}

\author{
Yi-Kun Zhao $\mathbb{D},{ }^{1}$ Guo-Qing Wang $\mathbb{D}^{2},{ }^{2}$ Xiao-Xiao Zhan $\mathbb{D}^{3},{ }^{3}$ and Peng-Hui Yang $\mathbb{D}^{4}$ \\ ${ }^{1}$ Art College, Anhui University of Finance and Economics, Bengbu 233030, China \\ ${ }^{2}$ School of Finance, Anhui University of Finance and Economics, Bengbu 233030, China \\ ${ }^{3}$ School of Law, Anhui University of Finance and Economics, Bengbu 233030, China \\ ${ }^{4}$ School of Statistics and Applied Mathematics, Anhui University of Finance and Economics, Bengbu 233030, China
}

Correspondence should be addressed to Peng-Hui Yang; yangpenghui666@163.com

Received 21 April 2021; Accepted 10 May 2021; Published 19 May 2021

Academic Editor: Huihua Chen

Copyright (C) 2021 Yi-Kun Zhao et al. This is an open access article distributed under the Creative Commons Attribution License, which permits unrestricted use, distribution, and reproduction in any medium, provided the original work is properly cited.

\begin{abstract}
This paper makes a quantitative analysis of the comprehensive influence of music networks. Firstly, 11 music features are selected from energy, popularity, and other aspects to build a comprehensive evaluation index of music influence, and the PageRank algorithm is used to quantify the music influence. Secondly, the multiobjective logistic regression is used to construct the music similarity measurement model and, combined with music influence and music similarity, to judge whether the influence of different musicians is the actual influence. Thirdly, the influence and similarity of the same music genre and different music genres are analyzed by using the two-way cluster analysis method. Finally, the lasso region is used for feature selection to obtain the change factors in the process of music evolution and analyze the dynamic changes in the process of music development. Therefore, this paper uses network science to build a dynamic network to analyze the similarity of music, the evolution process, and the impact of music on culture, which has certain research significance and practical value in the fields of music, history, social science, and practice.
\end{abstract}

\section{Introduction}

As an important part of human civilization, music has a profound impact on the development of human society. In the process of creating music, artists are influenced by many factors, such as other musicians, music schools, music genres, social events, and political events. In turn, the continuous creation of musicians also promotes the development of music. In recent years, scholars try to use machine learning and deep learning model to explore the impact of music development. According to our task and preliminary thinking, we searched and read articles about music genre classification and recognition, music impact evaluation, and so on. In the research content related to music genre classification, it mainly analyzes from the perspective of single music influence. Some literature considers the perspective of music genre classification, and on this basis, it has better practical significance to evaluate the music influence.

Naser and Saha [1] analyzed the influence of music on emotion and creativity by establishing an egg model. Banerjee et al. [2] used deterministic and nondeterministic methods to study the influence of different music. In the analysis of seriousness, this paper analyzes the music faction from two dimensions of acoustics and music characteristics [3]. Suganda et al. [4] and Li et al. [5] constructed a deep neural network model for music classification by using the spectrum, evaluated and combined the concept of fusion segment feature proposed by Dai et al. [6], verified the effectiveness of music feature segmentation and extraction, and introduced long-term short-term memory (LSTM) into the field of music genre recognition. Sim et al. [7] used the multilevel MRA artificial neural network method to understand and predict 
the relationship between music influence and music influence. On the basis of continuous integration of traditional filtering method and packing method, various new search algorithms and evaluation criteria for classifiers are constantly changing, such as neural network pruning method [8] and fuzzy entropy evaluation of feature set [9], which provide a good idea for support vector machine [10] and Gaussian mixture model [11]. Burred and Lerch [12] classified music types based on crnn algorithm. Cheng et al. [13] analyzed the advantages of the music genre in intensity tests. Nilson et al. [14] studied the analysis of the music emotion recognition method based on data features and used Li's [15] LDA model to process music tags, and weighted processing improved the accuracy of classification. Zhen et al. [16] input MFCC features into the KNN model and conducted experiments on gtzan dataset [17], which verified the effectiveness of the KNN model in music genre recognition. Tzanetakis and Cook [18] compared the ability of the gradient enhancement model and the additional tree model to extract multidimensional digital features of music types and proved that the two models can effectively identify the feature information of music types. The Gauss-Seidel method proposed by Donald Goldfarb [19] and the basic principle and idea of clustering proposed by Goldfarb et al. [20] also provide ideas for the creation of music impact indicators.

This paper constructs a comprehensive and multidimensional music influence network and, on this basis, constructs a music similarity measurement model and discusses the influence of music, the similarity of music schools, and the dynamic changes of music in detail. It mainly includes the following aspects: creating music influence network: PageRank is used to construct a directed network to reveal the types of interaction between musicians and music; research on constructing music similarity measurement model: the supervised classification model is used to calculate the music similarity of samples; bidirectional clustering: analysis of the similarity and influence between and within genres; and lasso regression: using lassos to identify indicators that significantly explain the dynamic factors and explain how genres or artists change over time. The existing model is analyzed, and a general model considering a variety of data is proposed. The overall idea is shown in Figure 1.

\section{Data Sources and Basic Assumptions}

The data of this paper comes from question $D$ of the 2021 American college students' mathematical modeling contest. In order to facilitate the solution of the problem, we propose the following assumptions:

(i) We assume that the average characteristics of all music created by a musician in a year can represent the musical characteristics of a musician throughout the year

(ii) We assume that the average characteristics of all musicians in a year can represent the musical characteristics of the whole year

(iii) We assume that missing data does not play an important role in the model (iv) We assume that there is no deviation in the internal relationship between the indicators in the model

\section{Exploratory Data Analysis}

When we get the preliminary data set, we first use the normalization method to process the data, evaluate the existing data information through numerical processing and comparison, and mine the correlation and change trend between the data, so as to help us understand the original data set to the maximum extent. Here is what we found.

3.1. Exploration of Data Loss Value. Through the analysis of the data sets, we found that there is no missing value in all the data sets, but there is some information asymmetry between the data sets. Here is our analysis.

The musician with ID 477787 has no information in "data_by_artist", so we deleted it. Some musicians in "data_by_artist", do not have data in "influence_data", and they lack information about the year and genre of influence. Considering a large amount of information about musicians, few musicians lack information. In order to facilitate subsequent analysis and modeling, we extract these musicians' data from "data_by_artist", After deletion, we still have the data of 5602 musicians. At the same time, there are many people who make up a small proportion of music. For the convenience of analysis, we also delete them. After the deletion, we still have 91731 music data.

3.2. Data Standardization. Sometimes, due to the needs of the model, we need to standardize the continuous variables in the data before modeling, that is, using the following formula:

$$
\frac{x_{i j}-\bar{x}_{\cdot j}}{s_{\cdot j}},
$$

where $x_{i j}$ is the element in rows $i$ and $j$ in the dataset, $\bar{x}_{. j}$ is the average of column $j$, and $s_{. j}$ is the standard deviation of column $j$.

3.3. Variable Analysis. We calculate the mean, median, quantile, maximum, and minimum values of continuous variables in "full_music_data", The results are shown in Table 1.

Discrete variables can be divided into explicit variables, pattern variables, and key variables. Among them, there are 88361 explicit variables of type 0 and 3370 variables of type 1 . The number of type 0 variables is nearly 26 times that of type 1 variables. According to experience, we can delete this variable in future analysis. There are 25324 explicit variables in Category 0, 66407 explicit variables in Category 1, and 12 key variables in Category 1 , which are expressed in the form of a histogram, as shown in Figure 2.

As can be seen from Figure 2, in "full_music_data", there are more categories 0,2 , and 9 and less categories 3 and 6 in the data set. The above is an exploratory analysis of each data set. In the next model, we will select variables and observations according to the actual situation of the data set, the 


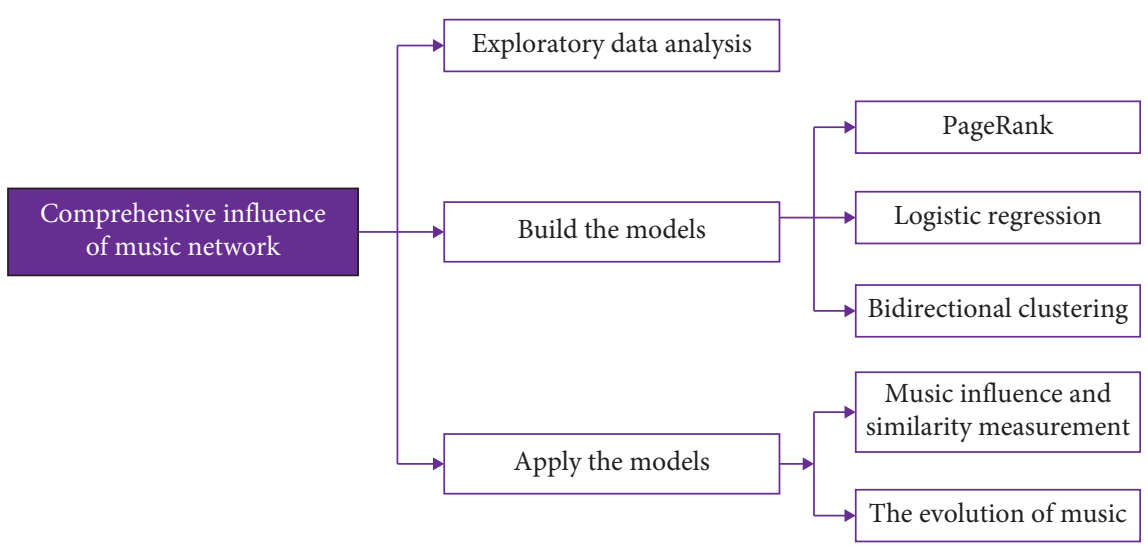

Figure 1: Flow diagram.

TABLE 1: Descriptive statistics of continuous variables.

\begin{tabular}{|c|c|c|c|c|c|c|}
\hline Value & Danceability & Energy & Valence & Tempo & Loudness & Acousticness \\
\hline Min & 0.01 & 0.01 & 0.12 & 30.0 & --60.00 & 0.03 \\
\hline 1st $\mathrm{Qu}$ & 0.42 & 0.33 & 0.33 & 96.52 & -13.40 & 0.05 \\
\hline Median & 0.54 & 0.55 & 0.54 & 117.21 & -10.00 & 0.34 \\
\hline Mean & 0.52 & 0.54 & 0.54 & 119.34 & -10.59 & 0.41 \\
\hline 3rd Qu & 0.64 & 0.76 & 0.75 & 137.61 & -6.94 & 0.76 \\
\hline Max & 0.99 & 1.00 & 1.00 & 244.09 & 3.74 & 1.00 \\
\hline Value & Instrumentalness & Liveness & Speechiness & Duration_ms & Popularity & 一 \\
\hline Min & 0.02 & 0.03 & 0.01 & 5108 & 0.02 & - \\
\hline 1st $\mathrm{Qu}$ & 0.10 & 0.10 & 0.03 & 175502 & 25.00 & - \\
\hline Median & 0.09 & 0.13 & 0.04 & 218600 & 36.00 & - \\
\hline Mean & 0.12 & 0.20 & 0.06 & 237414 & 35.91 & - \\
\hline 3rd Qu & 0.03 & 0.26 & 0.06 & 272640 & 48.00 & - \\
\hline Max & 1.00 & 1.00 & 0.96 & 3816373 & 100.0 & - \\
\hline
\end{tabular}

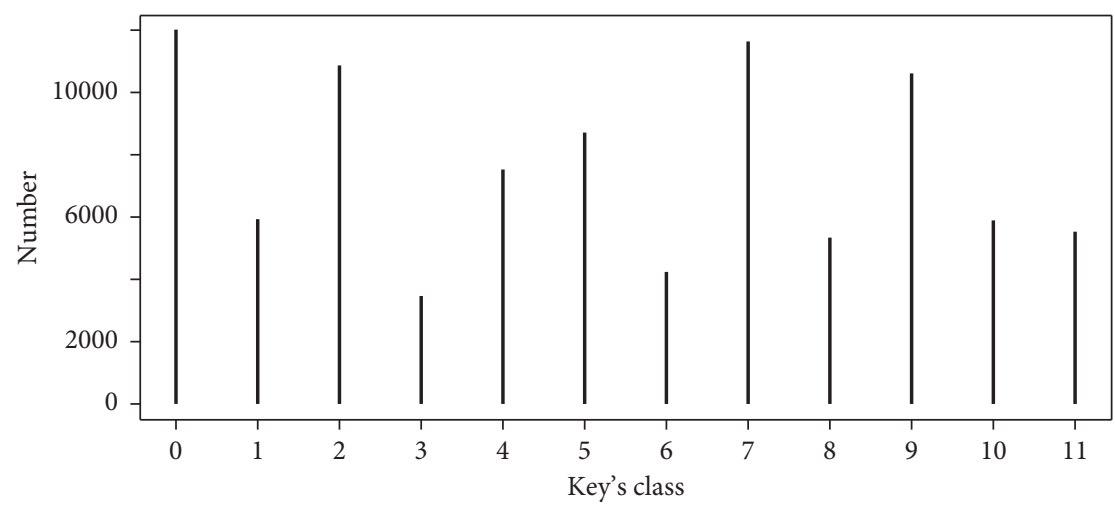

Figure 2: The number of categories histogram of the key variable.

content of the problem, the background knowledge, and the application scope of the model.

\section{Quantitative Ranking of Music Influence Network Based on PageRank Algorithm}

4.1. PageRank Principles. PageRank is a search engine technology based on hyperlinks. According to the links between the nodes, the importance of the contact is divided from 0 to 10 columns, and 10 points is the full score. A high
PR value indicates that the node is very important [21]. PageRank schematic is shown in Figure 3.

4.2. Calculating Music Influence. In this paper, the importance of influencers in the network is sorted by using the improved PageRank algorithm [22, 23], and the influence of musicians is calculated. For example, for the musicians' influencers and followers, PageRank's principles are as follows. 

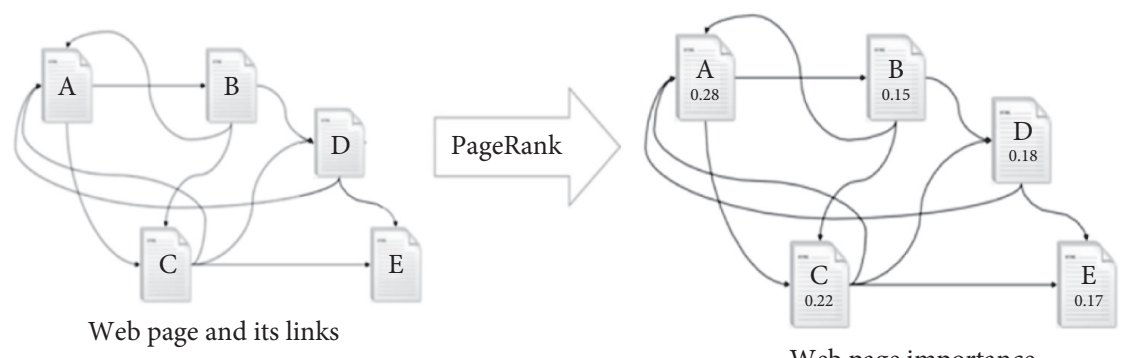

Figure 3: PageRank schematic.

First, we construct a dimension adjacency matrix by the following relations among $n$ musicians:

$$
M=\left[\begin{array}{cccc}
0 & 1 & \cdots & 1 \\
0 & 0 & \cdots & 0 \\
\vdots & \vdots & \ddots & \vdots \\
1 & 1 & \cdots & 0
\end{array}\right]
$$

If the values of $i$ row and $j$ column in the matrix are equal to 1 , then musician $j$ has an influence on musician $i$. On the contrary, if the values of row $i$ and column $j$ in the matrix are equal to 0 , then musician $j$ has no effect on musician $i$.

To avoid divergence, we normalize the columns of the matrix so that the sum of each column in the matrix equals 1 :

$$
M=\left[\begin{array}{cccc}
0 & 1 & \cdots & 1 \\
0 & 0 & \cdots & 0 \\
\vdots & \vdots & \ddots & \vdots \\
1 & 1 & \cdots & 0
\end{array}\right] \times\left[\begin{array}{c}
\frac{1}{\sum\left(M_{\cdot 1}\right)} \\
\vdots \\
\frac{1}{\sum\left(M_{\cdot N}\right)}
\end{array}\right] .
$$

In order to avoid the algorithm convergence failure due to the dead chain of the algorithm, we jump along the connection relationship between musicians with a certain probability. We jump along the connection between musicians with a certain probability $\beta$ and jump to any musician with a certain probability $1-\beta$. To describe it in mathematical language is to construct a matrix $\widehat{M}$.

$$
\widehat{M}=\beta * M+(1-\beta) *\left(\frac{1}{N}\right)_{N * N},
$$

where $(1 / N)_{N \times N}$ is the $N \times N$ dimension adjacency matrix with all internal elements $(1 / N)$. We set the $\beta=0.85$. Finally, we initialize the influence degree vector of $N \times 1$ dimension; that is, $r^{(0)}=\operatorname{random}\left(r_{1}, r_{2}, \ldots, r_{N}\right)$, where $r_{i} \in(0,1)$, and through the iteration of the following formula until convergence, we get the final influence vector $r^{(t+1)}$.

$$
r^{(t+1)}=\widehat{M} \times r^{(t)}
$$

4.3. Analysis of Calculation Results. We used the PageRank formula to calculate the importance and got the influence of 5602 musicians. The results are shown in Table 2 .

From Table 2, we find that musicians with ID 36106, 3495279 , and 3480388 are the three most influential musicians. 1960, 2010, and 2010 are the first ten years of their music career, respectively. Pop/Rock musicians make up $75 \%$ of the top 20 , and Latin musicians make up 15\%. From the time when musicians began to write, $50 \%$ of them started to write in 2010 and $25 \%$ in 2000 , and $75 \%$ of the top 20 musicians started to write in the 21 st century.

In order to further explore the relationship between the influence, genre, and year of musicians in the target network, we conducted an in-depth analysis of influential musicians among 5062 musicians. The influence of the 200th most influential musician accounts for $10.12 \%$ of the first most influential musician. Based on the assessment of musicians' influence in previous studies, we defined the top $200 \mathrm{mu}-$ sicians as influential. For selected influential musicians, we compared their music genres and the time they started their careers. The results are shown in Figures 4 to 5 .

From Figure 4, we find that the most influential music genre is pop/rock, accounting for $65.5 \%$, followed by country music, electronic music, and R\&B music, accounting for $11.5 \%, 5 \%$, and $5 \%$, respectively. In terms of the time when musicians start their careers, $45.5 \%$ of them start their careers in 2010 and $21.5 \%$ in 2020 , which means that $67 \%$ of the most influential musicians start their careers in the early 21 st century. In 1990, $26.5 \%$ of musicians began to work in music, and in 1980, 4.5\% began to work in music. By the end of the 20th century, $31 \%$ of the musicians began to write music. Based on the above analysis, we can draw a preliminary conclusion: since the end of the 20th century, influenced by economic and social stability, scientific and cultural development, and other factors, pop/rock, country, electronics, $\mathrm{R} \& \mathrm{~B}$, and other music schools have developed rapidly, and the number and influence of musicians of these schools have increased.

\section{Music Similarity Measurement Model}

5.1. The Principle of Supervised Classification Pattern. When the response variable is binary, the logistic regression [24] model is usually used. The response variable is represented by $G$, where $G=\{1,2\}$, and takes the value in the label of the element which is arbitrary. The logistic 
TABLE 2: Top 20 influential musicians.

\begin{tabular}{lcccc}
\hline artist_name & artist_id & main_genre & Power & Year \\
\hline Steve miller band & 36106 & Pop/Rock & $8.26 E-42$ & 1960 \\
CNCO & 3480388 & Latin & $6.91 E-42$ & 2010 \\
Andy Black & 3495279 & Pop/Rock & $6.17 E-42$ & 2010 \\
Spin Doctors & 10412 & Pop/Rock & $5.74 E-42$ & 1980 \\
The Oh Hellos & 3366296 & Pop/Rock & $5.59 E-42$ & 2010 \\
Cheat Codes & 3391646 & Electronic & $4.20 E-42$ & 2010 \\
Fifth Harmony & 3145706 & Pop/Rock & $3.67 E-42$ & 2010 \\
One direction & 2766592 & Pop/Rock & $3.56 E-42$ & 2010 \\
The Lumineers & 2873356 & Pop/Rock & $3.16 E-42$ & 2000 \\
Ed Sheeran & 2639628 & Pop/Rock & $3.05 E-42$ & 2000 \\
Gnash & 3462822 & Pop/Rock & $2.97 E-42$ & 2010 \\
Collie Buddz & 476599 & Reggae & $2.88 E-42$ & 2000 \\
Chino \& Nacho & 1471849 & Latin & $2.49 E-42$ & 2000 \\
Juanes & 245250 & Latin & $2.42 E-42$ & 1980 \\
James TW & 3451786 & Pop/Rock & $2.39 E-42$ & 2010 \\
Matt Nathanson & 385797 & Pop/Rock & $2.37 E-42$ & 1990 \\
John Mayer & 239827 & Pop/Rock & $2.33 E-42$ & 1990 \\
With Confidence & 3445208 & Pop/Rock & $2.23 E-42$ & 2010 \\
Christina Perri & 2508103 & Pop/Rock & $2.21 E-42$ & 2000 \\
Cloves & 3460695 & Pop/Rock & $2.20 E-42$ & 2010 \\
\hline
\end{tabular}

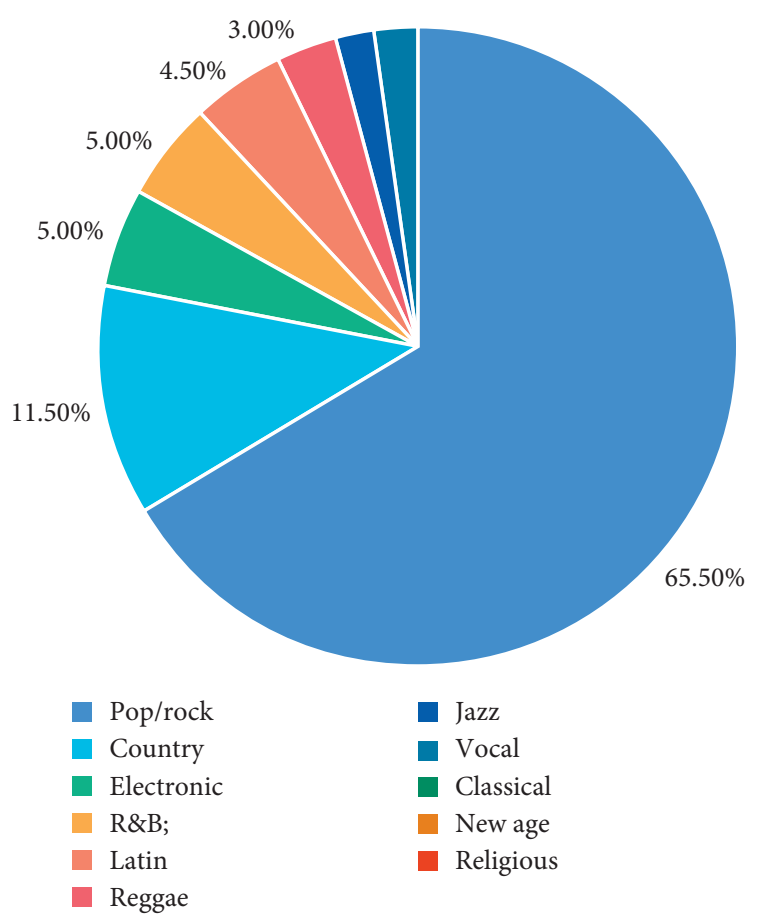

FIGURE 4: The proportion of each genre.

regression model uses the linear function of the predictor to express class conditional probability:

$$
\begin{gathered}
\operatorname{Pr}(G=1 \mid x)=\frac{1}{1+e^{-\left(\beta_{0}+x^{T} \beta\right)}}, \\
\operatorname{Pr}(G=2 \mid x)=\frac{1}{1+e^{+\left(\beta_{0}+x^{T} \beta\right)}}=1-\operatorname{Pr}(G=1 \mid x),
\end{gathered}
$$

or the logarithmic formula of quasi-conditional probability:

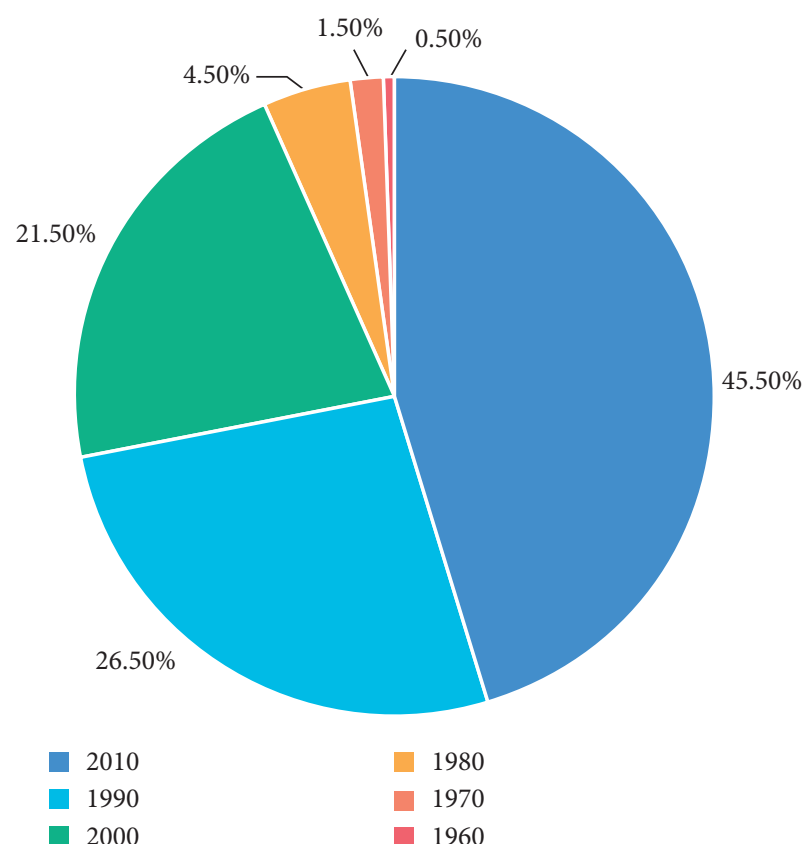

FIgURE 5: The proportion of music career beginning year.

$$
\log \frac{\operatorname{Pr}(G=1 \mid x)}{\operatorname{Pr}(G=2 \mid x)}=\beta_{0}+x^{T} \beta
$$

The linear logistic regression model can be extended to a multivariate logistic model when the classification response variable $G$ is at the level of $k>2$. The traditional method is to extend the logarithm formula of the conditional probability of binary logarithm regression model to logarithm $k-1$.

$$
\log \frac{\operatorname{Pr}(G=\ell \mid x)}{\operatorname{Pr}(G=K \mid x)}=\beta_{0 \ell}+x^{T} \beta_{\ell}, \quad \ell=1, \ldots, K-1 .
$$

This is the dimension vector of the coefficient $p$. We chose a more symmetrical approach. Our model is as follows:

$$
\operatorname{Pr}(G=\ell \mid x)=\frac{e^{\beta_{0 \ell}+x^{T} \beta_{\ell}}}{\sum_{k=1}^{K} \beta_{0 k}+x^{T} \beta_{k}} .
$$

If there are no constraints, the parameterization is not estimable. Therefore, we regularize the maximum likelihood (polynomial) fitting model. In other words, let $p_{\ell}\left(x_{i}\right)=\operatorname{Pr}\left(G=\ell \mid x_{i}\right)$ and $g_{i} \in\{1,2, \ldots, K\}$ be the $i$-th response; we estimate the parameters by maximizing the probability of penalty logarithm.

$$
\max _{\left\{\beta_{0 \ell}, \beta_{\ell}\right\}_{1}^{K} \in \mathbb{R}^{K(p+1)}}\left[\frac{1}{N} \sum_{i=1}^{N} \log p_{g_{i}}\left(x_{i}\right)-\lambda \sum_{\ell=1}^{K} P_{\alpha}\left(\beta_{\ell}\right)\right],
$$

where $y$ is the indicator response matrix of dimension $N K$, and the elements are

$$
y_{i l}=I\left(g_{i}=l\right) .
$$


5.2. Construction of Music Similarity Model. We use the multiobjective logistic model [25] to build the music similarity index, and the specific process is as follows:

(i) Taking 20 music genres as dependent variables and music characteristics as independent variables, a multiobjective logistic model is constructed to obtain the probability value of each music in 20 music genres. Then, we select the $K$ genres with the highest probability among the genres that each music is most likely to belong to $\mathrm{cp}_{i}=\left\{\mathrm{cp}_{i 1}, \mathrm{cp}_{i 2}, \ldots, \mathrm{cp}_{i 20}\right\}, i=$ $1,2, \ldots, N$, and we set $k=6$; that is, $C_{i}=\left\{c_{i 1}, c_{i 2}\right.$, $\left.c_{i 3}, c_{i 4}, c_{i 5}, c_{i 6}\right\}, i=1,2, \ldots, N$.

(ii) We get the intersection $\left(C_{i}, C_{j}\right), i=1,2, \ldots, N, j=$ $1,2, \ldots, N$, of music in six music genres and calculate the number of elements in the intersection num $_{i j}=$ length (intersection $\left(C_{i}, C_{j}\right)$ ), and finally, we get the similarity $s_{i j}=\left(\right.$ num $\left._{i j} / k\right)$.

This view holds that each music genre has its own music characteristics, so it is easy to think that each music genre can be regarded as a complete musical feature. If the two kinds of music are very similar and their music characteristics should be very similar, the first six music types with the highest probability obtained by the logistic model should have high similarity coincidence. We take two songs, Ernie and Wandering Eye, by musician 178301 as an example. Through multiobjective logistic analysis, the first six types with the highest probability are shown in Table 3.

So, their similarity is 1 . It can be said that the similarity of these two pieces of music is still very high.

In addition, we can also use a logistic background music similarity measurement model to calculate the similarity between musicians. As shown in the left figure of Figure 6, the deeper the color expression among different musicians, the closer their music features are, which means that the higher the similarity between their music.

5.3. Case Analysis. The music characteristics of different musicians affect the distance between musicians, which also affects the similarity between musicians. So, must musicians in a genre be more similar than cross-genre musicians? In order to study this problem, we randomly selected 100 musicians from 5062 musicians and analyzed the similarities between the same type of musicians and different types of musicians. The results are shown in the right figure in Figure 7.

The following can be seen from the similarity value between different genres in Figure 7: generally speaking, the music similarity within a unified genre is generally higher than that between different genres. The music similarity between the same genre is more than 0.6 , and the selection of similarity up to 1 is due to the small sample size, which leads to the small number of musicians extracted from a specific genre. It can be solved by enlarging the sample. For some genres, they are more similar to some genres, even higher than the similarity within this genre. For example, the similarity between vocal music musicians and country music
TABLE 3: Music corresponds to the most probable faction.

\begin{tabular}{lcccccc}
\hline Music name & \multicolumn{5}{c}{ The top six factions with the highest } \\
& probability \\
\hline Ernie & 6 & 8 & 11 & 14 & 15 & 16 \\
Wandering eye & 6 & 8 & 11 & 14 & 15 & 16 \\
\hline
\end{tabular}

musicians is 0.65 , which is greater than that between country music musicians by 0.64 . The reason for this phenomenon can initially speculate that the popularity of vocal music schools is earlier than that of country music, and the musical characteristics of vocal music genres have a certain foundation. In the development of country music, the vocal music genre can get more references from the music style of the vocal music genre, and they have high similarity.

\section{Analysis of Music Schools}

6.1. Bidirectional Clustering Principle. The purpose of bidirectional clustering [26] is to find the submatrix satisfying the condition in the gene expression data matrix, so that the expression of the feature set in the submatrix is consistent on the corresponding observation set. This is similar to the best subset selection in the regression problem. Just as the best subset selection problem is successfully solved by solving the convex surrogate problem (lasso), we will use the convex relaxed combination problem to select the row and column partitions. Then, the bidirectional clustering model is equivalent to the chessboard average model, which is exhaustive because each matrix element is assigned to a bidirectional $\omega_{i j}=\omega_{j i}$ clustering. This is different from other bidirectional clustering models, which identify possible overlapping row and column subsets, but not exhaustive. The parameter estimation of the chessboard model includes partition and the average value of each partition.

\subsection{Bidirectional Clustering Process.}

We determine the partition by minimizing the following convex criteria:

$$
F_{\gamma}(U)=\frac{1}{2}\|X-U\|_{F}^{2}+\gamma\left[\Omega_{W}(U)+\Omega_{\bar{W}}\left(U^{T}\right)\right]
$$

where $J(U)=\Omega_{W}(U)+\Omega_{\widetilde{W}}\left(U^{T}\right), \quad \Omega_{W}(U)=\sum_{i<j} w_{i j} \| U_{\cdot i}-$ $U_{. j 2} \|$, and $U_{i}\left(U_{i}\right.$ ) represents the $i$-th column (row) of a matrix. The quadratic term quantifies the approximation degree of $U$ to $X$, and the regular term penalizes the deviation from the chessboard pattern. Parameters $\gamma \geq 0$ adjust the trade-off between the two terms and $\widetilde{\omega}_{i j}=\widetilde{\omega}_{j i}$.

In this paper, the alternating minimization algorithm (AMA) proposed by Chi Eric and Lange [27] is used to solve the convex clustering problem. With the increase of penalty coefficient from small to large $(1,5.62,31.62177 .82)$, we get the clustering process in Figures $8(\mathrm{a})-8(\mathrm{~d})$.

It can be seen that with the increase of penalty coefficient, the $U$ matrix of reconstructed $x$ gradually presents the overall pattern of the chessboard. Next, we only look at the clustering results with penalty coefficient of. 


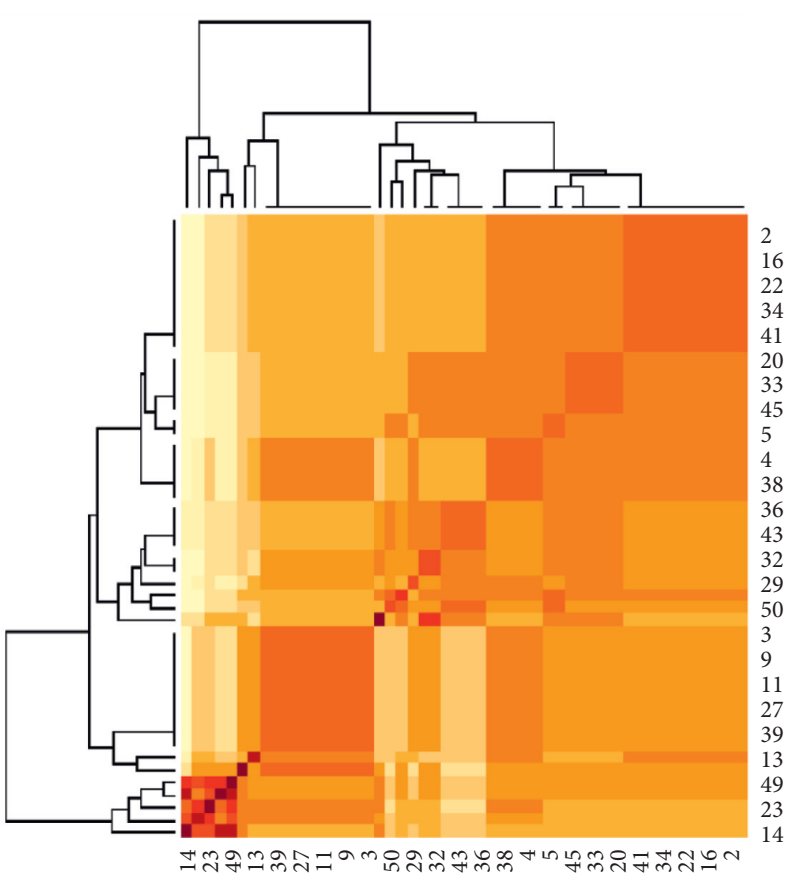

(a)

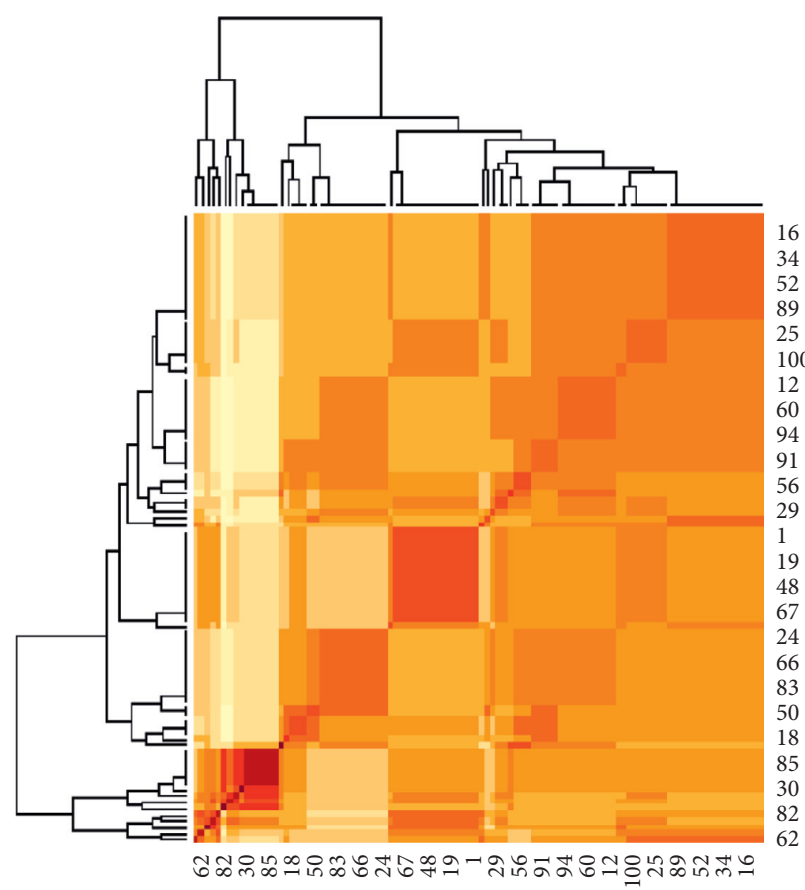

(b)

Figure 6: Musician similarity graph.

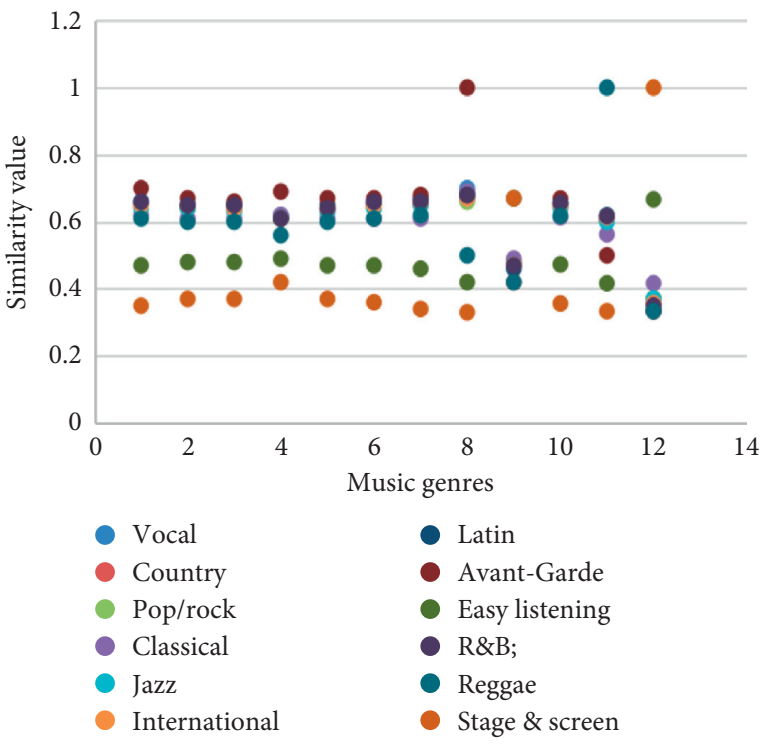

FIgURE 7: Similarity between music genres.

6.3. Result Analysis of Bidirectional Clustering. Based on loudness, valence, energy, tempo, danceability, speechiness, liveliness, acousticness, duration, and instrumentalness, the classification of 20 different music schools is shown in Figure 9.

According to the results of bidirectional clustering, 20 different music genres are divided into 5 groups. According to the comparison of rhythms, the similarities and differences of the five music characteristic values of Latin, country, children, reggae, blues, pop/rock, $R \& B$, electronics, and religion all have good rhythm and positive vitality. There are obvious similarities among international, folk, and vocal music, all of which have a certain sense of rhythm and moderate overall loudness. Through the comparison of sound, duration, and musical instruments, we can see that there are obvious similarities among classical, new era, stage, and screen music schools, with similar repertoire and a higher degree of musical instruments. Similarly, easy listening is very similar to jazz in that there are specific vocals in their repertoire. Comedy/spoken English is divided into groups. Among the 19 music schools, only comedy/spoken language has positive values of discourse and liveliness, which indicates that comedy/spoken language is a music school of speaking or reciting poetry for the audience.

\section{Influence of Followers}

7.1. The Screening Principle of Actual Influence. A musician can list a dozen or more musicians who have an impact on them. ICM provides us with a data set, including the impact on their relationship with their followers. But do these influential musicians really influence the music that their followers make? In order to further explore the influence of influencers on followers, we use the data set of the relationship between influencers and followers, and the similarity matrix between musicians to construct a matrix that can filter out the actual influence.

Firstly, a $0-1$ matrix $M$ of $5602 \times 5602$ is constructed by using the data set, where 0 represents no relationship between the two musicians and 1 represents the relationship between the two musicians. For example, we can set the $M$ as follows: 


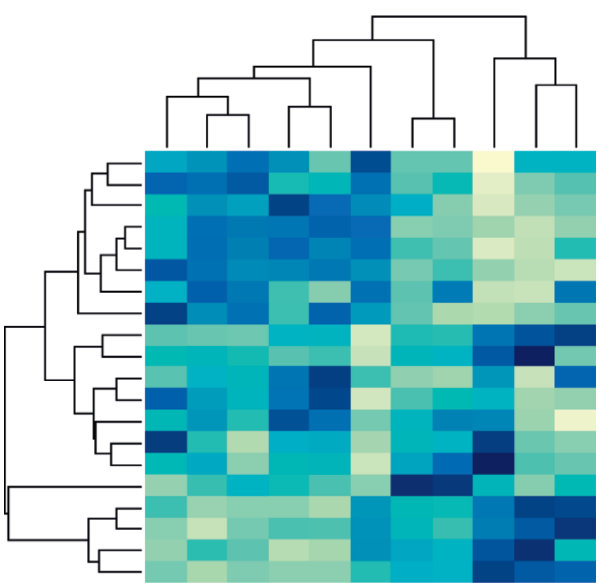

(a)

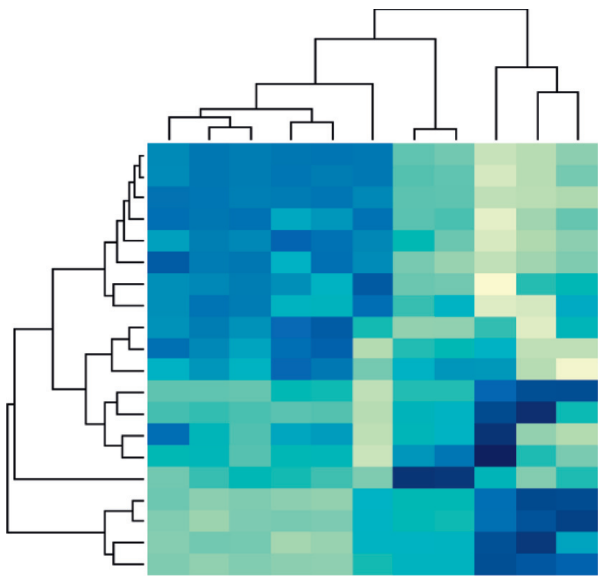

(c)

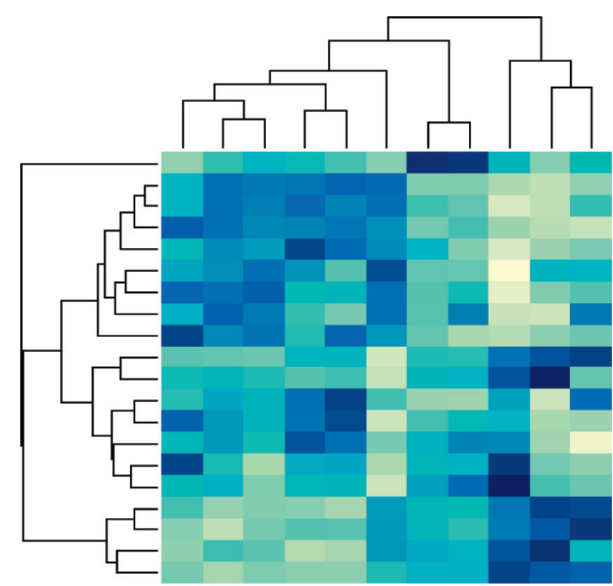

(b)

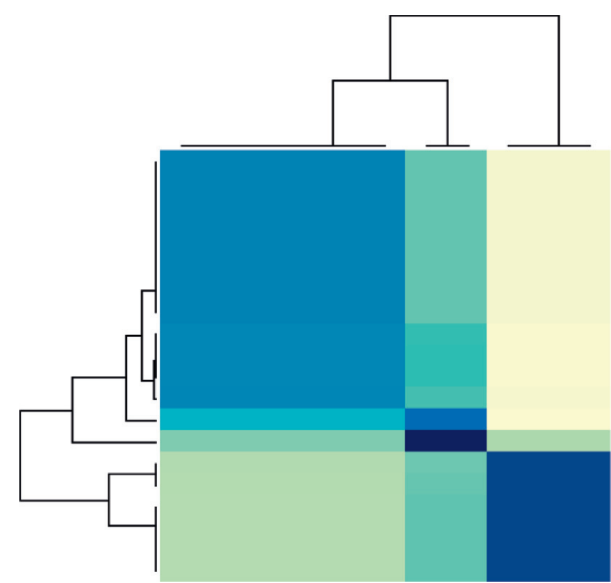

(d)

Figure 8: Clustering process. (a) Step 1. (b) Step 2. (c) Step 3. (d) Step 4.

\begin{tabular}{|c|c|c|c|c|c|c|c|c|c|c|}
\hline & Loudness & Valence & Energy & Tempo & Danceability & Speechiness & Liveness & Acousticness & Duration_ms & Instrumentalness \\
\hline Latin & 0.316 & 0.316 & 0.316 & 0.316 & 0.316 & 0.025 & 0.025 & -0.257 & -0.257 & -0.257 \\
\hline Country & 0.316 & 0.316 & 0.316 & 0.316 & 0.316 & 0.025 & 0.025 & -0.257 & -0.257 & -0.257 \\
\hline Children's & 0.316 & 0.316 & 0.316 & 0.316 & 0.316 & 0.025 & 0.025 & -0.257 & -0.257 & -0.257 \\
\hline Reggae & 0.316 & 0.316 & 0.316 & 0.316 & 0.316 & 0.025 & 0.025 & -0.257 & -0.257 & -0.257 \\
\hline Blues & 0.316 & 0.316 & 0.316 & 0.316 & 0.316 & 0.025 & 0.025 & -0.257 & -0.257 & -0.257 \\
\hline Pop/rock & 0.316 & 0.316 & 0.316 & 0.316 & 0.316 & 0.025 & 0.025 & -0.257 & -0.257 & -0.257 \\
\hline $\mathrm{R} \& \mathrm{~B}$ & 0.316 & 0.316 & 0.316 & 0.316 & 0.316 & 0.025 & 0.025 & -0.257 & -0.257 & -0.257 \\
\hline Electronic & 0.316 & 0.316 & 0.316 & 0.316 & 0.316 & 0.025 & 0.025 & -0.257 & -0.257 & -0.257 \\
\hline Religious & 0.316 & 0.316 & 0.316 & 0.316 & 0.316 & 0.025 & 0.025 & -0.257 & -0.257 & -0.257 \\
\hline International & 0.245 & 0.245 & 0.245 & 0.245 & 0.245 & 0.018 & 0.018 & -0.201 & -0.201 & -0.201 \\
\hline Folk & 0.216 & 0.216 & 0.216 & 0.216 & 0.216 & 0.019 & 0.019 & -0.192 & -0.192 & -0.192 \\
\hline Vocal & 0.117 & 0.117 & 0.117 & 0.117 & 0.117 & 0.016 & 0.016 & -0.141 & -0.141 & -0.141 \\
\hline Comedy/spoken & 0.429 & 0.429 & 0.429 & 0.429 & 0.429 & 1.393 & 1.393 & 0.364 & 0.364 & 0.364 \\
\hline Easy listening & -0.355 & -0.355 & -0.355 & -0.355 & -0.355 & -0.171 & -0.171 & 0.270 & 0.270 & 0.270 \\
\hline Jazz & -0.355 & -0.355 & -0.355 & -0.355 & -0.355 & -0.171 & -0.171 & 0.270 & 0.270 & 0.270 \\
\hline Avant-garde & -0.865 & -0.865 & -0.865 & -0.865 & -0.865 & -0.338 & -0.338 & 0.549 & 0.549 & 0.549 \\
\hline Stage \& screen & -0.865 & -0.865 & -0.865 & -0.865 & -0.865 & -0.338 & -0.338 & 0.549 & 0.549 & 0.549 \\
\hline New age & -0.865 & -0.865 & -0.865 & -0.865 & -0.865 & -0.338 & -0.338 & 0.549 & 0.549 & 0.549 \\
\hline Classical & -0.865 & -0.865 & -0.865 & -0.865 & -0.865 & -0.338 & -0.338 & 0.549 & 0.549 & 0.549 \\
\hline
\end{tabular}

FIGURE 9: Similarities and influences between genres (different clusters) and within genres (same clusters). 


$$
M=\left[\begin{array}{cccc}
0 & 1 & \cdots & 1 \\
1 & 0 & \cdots & 1 \\
\vdots & \vdots & \ddots & \vdots \\
0 & 0 & \cdots & 1
\end{array}\right] .
$$

Then, the similarity matrix between 5602 musicians is used to construct the 0-1 matrix $P$; that is, when the elements in the similarity matrix between musicians are greater than the threshold, the corresponding position element of the $P$ matrix is taken as 1; otherwise, when they are not greater than the threshold, the corresponding position element of the $P$ matrix is taken as 0 . Combined with previous studies,
TABLE 4: Musicians who really influenced Johnny Cash.

\begin{tabular}{lc}
\hline artist_id & artist_name \\
\hline 190946 & Lefty Frizzell \\
815039 & John Lee Hooker \\
45057 & The Louvin Brothers \\
74970 & Bob Wills \\
662800 & Hank Snow \\
198901 & Webb Pierce \\
200775 & Ernest Tubb \\
163198 & Carl Smith \\
\hline
\end{tabular}

we set the threshold of similarity to 0.8 . For example, the transformation from similarity matrix to $P$ matrix is

$$
\text { similarity matrix }=\left[\begin{array}{cccc}
0.2 & 0.85 & \cdots & 0.5 \\
0.7 & 0.4 & \cdots & 1 \\
\vdots & \vdots & \ddots & \vdots \\
0.6 & 0.9 & \cdots & 0.95
\end{array}\right] \longrightarrow{ }^{\text {threshold }=0.8} P=\left[\begin{array}{cccc}
0 & 1 & \cdots & 1 \\
0 & 0 & \cdots & 1 \\
\vdots & \vdots & \ddots & \vdots \\
0 & 1 & \cdots & 1
\end{array}\right] .
$$

Finally, a new matrix is obtained by multiplying the corresponding elements of the 0-1 matrix and the matrix. The meaning of each element in the matrix is as follows: the superposition of the similarity and influence of two musicians, which we define as the actual influence. If there is no influence relationship between the two musicians, the actual influence is 0 . If there is an influence relationship between two musicians, the actual influence depends on whether the similarity between them is greater than the threshold. When the similarity is greater than the threshold, there is a real influence relationship between two musicians.

$$
T=M * P=\left[\begin{array}{ccccc}
m_{11} * p_{11} & m_{12} * p_{12} & \cdots & m_{1 N} * p_{1 N} & \\
m_{21} * p_{21} & m_{22} * p_{22} & \cdots & m_{2 N} * p_{2 N} & \\
\vdots & \vdots & & \ddots & \vdots \\
m_{N 1} * p_{N 1} & m_{N 2} * p_{N 2} & \cdots & m_{N N} * p_{N N}
\end{array}\right]=\left[\begin{array}{cccc}
t_{11} & t_{12} & \cdots & t_{1 N} \\
t_{21} & t_{22} & \cdots & t_{2 N} \\
\vdots & \vdots & & \vdots \\
t_{N 1} & t_{N 2} & \cdots & t_{N N}
\end{array}\right]
$$

where $N=5602$.

7.2. Case Analysis. We randomly selected 5602 musicians and took the musician with ID 816890 (Johnny Cash) as an example. Based on the interaction and similarity data set between musicians with ID 816890 (Johnny Cash) and other musicians, the screening matrix of actual influence is constructed. We finally found the musicians who really influenced him, as shown in Table 4.

After getting the real influential musician ID 816890 (Johnny KASH), we further thought the following: are musicians who really influence Johnny KASH have more "infective" musical characteristics, or do they all play a similar role in affecting Johnny KASH's music? In order to explore this problem, we have done the following.

First, we get normalized data for musicians with ID 816890 (Johnny Cash) and the music characteristics that really affect his musicians, as shown in Table 5 .

Then, the sum of absolute distances of followers with ID 816890 on nine music features is calculated. For example, for the dance of musical features, we get the sum of absolute distances:

$$
|0.817-0.555|+|0.482-0.555|+\cdots+|0.372-0.555|=2.539 \text {. }
$$

For the nine features, the sum of the absolute distances calculated is shown in Figure 10.

Finally, look at Figure 10. We find that, compared with other musical features, the sum of absolute distances between acoustics and instrumentality is very small. Musical harmony is the two musical characteristics, and the musician with ID number 816890 has a real influence on these two characteristics.

\section{The Evolution of Music}

8.1. An Analysis of the Characteristics of Revolution. In the process of music evolution, some revolutionary changes in music characteristics may lead to a significant leap in music evolution $[28,29]$. So, which music characteristics are 
TABLE 5: Normalized data of music features.

\begin{tabular}{|c|c|c|c|c|c|c|}
\hline artist_name & artist_id & Danceability & Energy & Valence & Tempo & Loudness \\
\hline Lefty Frizzell & 190946 & 0.817 & -1.389 & 0.722 & -0.148 & -0.664 \\
\hline John Lee Hooker & 815039 & 0.482 & -1.135 & 0.469 & 0.294 & -1.322 \\
\hline The Louvin Brothers & 45057 & 0.372 & -1.370 & 0.655 & 0.098 & -0.293 \\
\hline Bob Wills & 74970 & 0.525 & -0.907 & 1.013 & 0.367 & -0.042 \\
\hline Hank Snow & 662800 & 0.208 & -0.619 & 0.798 & -0.256 & -0.506 \\
\hline Webb Pierce & 198901 & 0.340 & -0.830 & 1.193 & -0.611 & -0.142 \\
\hline Ernest Tubb & 200775 & 1.232 & -0.852 & 1.209 & 0.251 & -0.653 \\
\hline Carl Smith & 163198 & 1.307 & -0.697 & 2.037 & -0.942 & -0.327 \\
\hline Johnny cash & 816890 & 0.555 & -0.641 & 0.682 & -0.285 & -0.409 \\
\hline artist_name & artist_id & Acousticness & Instrumentalness & Liveness & Speechiness & - \\
\hline Lefty Frizzell & 190946 & 1.382 & -0.539 & -0.294 & -0.453 & - \\
\hline John Lee Hooker & 815039 & 1.147 & -0.415 & -0.354 & 0.238 & - \\
\hline The Louvin Brothers & 45057 & 1.222 & -0.564 & -0.286 & -0.496 & - \\
\hline Bob Wills & 74970 & 1.127 & -0.319 & 0.256 & -0.160 & - \\
\hline Hank Snow & 662800 & 1.425 & -0.014 & -0.079 & -0.458 & - \\
\hline Webb Pierce & 198901 & 1.505 & -0.554 & 0.460 & -0.514 & - \\
\hline Ernest Tubb & 200775 & 1.196 & -0.563 & -0.146 & -0.397 & - \\
\hline Carl Smith & 163198 & 1.127 & -0.564 & -0.995 & -0.374 & - \\
\hline Johnny Cash & 816890 & 1.176 & -0.466 & 0.405 & 0.378 & - \\
\hline
\end{tabular}

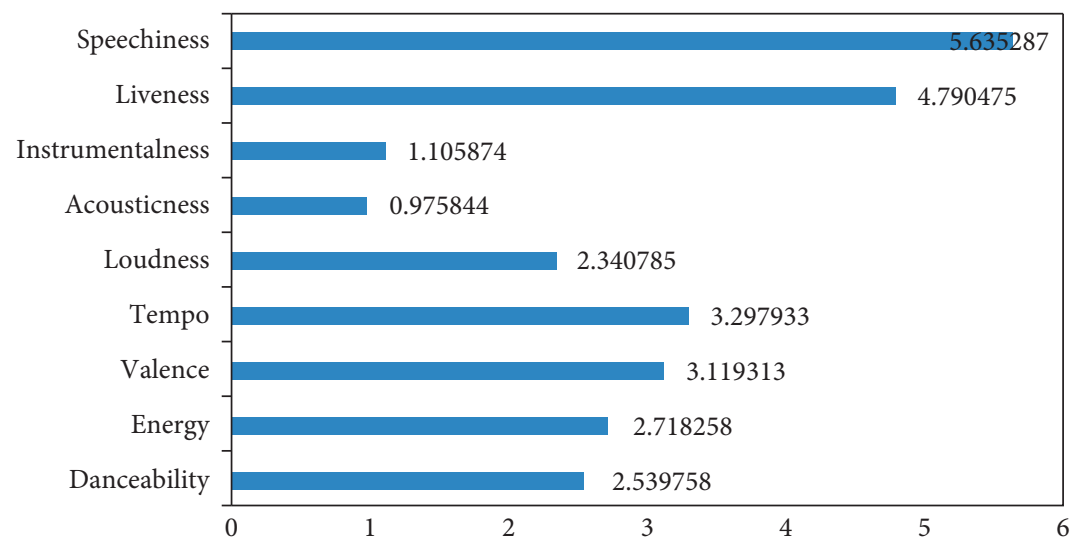

Figure 10: Sum of absolute distances.

revolutionary in the data? In order to find the revolutionary characteristics of music, we have done the following work.

We use the "data_by_year" data set to analyze the fluctuation of various music characteristics from 1921 to 2020. In order to eliminate the influence of the level of variable value and different measurement units on the measurement value of dispersion degree, we choose to use the dispersion coefficient to analyze the fluctuation of music characteristics. The calculation formula is as follows:

$$
v_{i}=\frac{s_{i}}{\bar{x}_{i}}
$$

We calculate the dispersion coefficients of the ten musical characteristics and set the threshold as 0.5 . Then, when the dispersion coefficient of a musical feature is greater than 0.5 , it will be revolutionary to a certain extent. The dispersion coefficient of each music feature is shown in Table 6.

From Table 6, we can see that the dispersion coefficient of acoustic, instrumental, and speech music features is greater than 0.5 , which is a revolutionary music feature.
8.2. The Impact of Major Changes. The characteristics of revolutionary music are often reflected in the songs created by musicians. So, in the directional network of musicians' influence, are there any musicians with significant influence who are the influencers of major changes? In order to find out, we have done the following work.

Firstly, we get the changing trend of acousticness, instrumentalness, and speechiness from 1921 to 2020.

From Figures 11-13, we find that acousticness changed greatly in 1926, 1927, 1929, 1930, and 1946. In 1924, 1929, and 1946, great changes took place in instrumentalness. In 1929, 1930, and 1935, speechiness changed a lot.

Secondly, we take the value of the three revolutionary music characteristics as the standard when they have changed greatly and look for the songs with the smallest absolute distance between the music characteristics and the standard value in the corresponding years. In 1924, 1926, $1927,1929,1930,1935$, and 1946, a total of 10 tracks with revolutionary application characteristics were found (as shown in Table 7). 


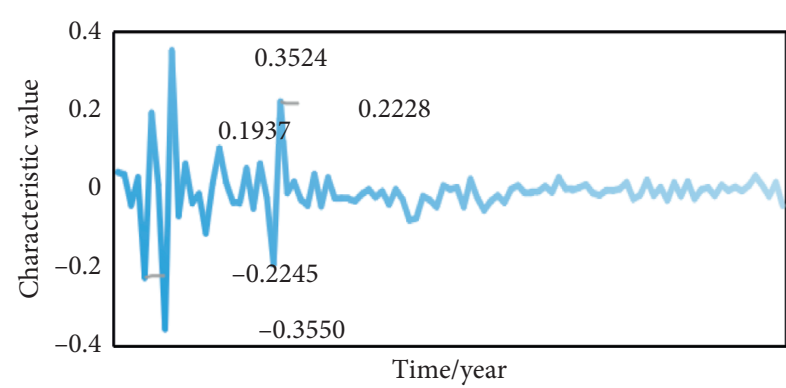

FIgURE 11: The changing trend of the acousticness.

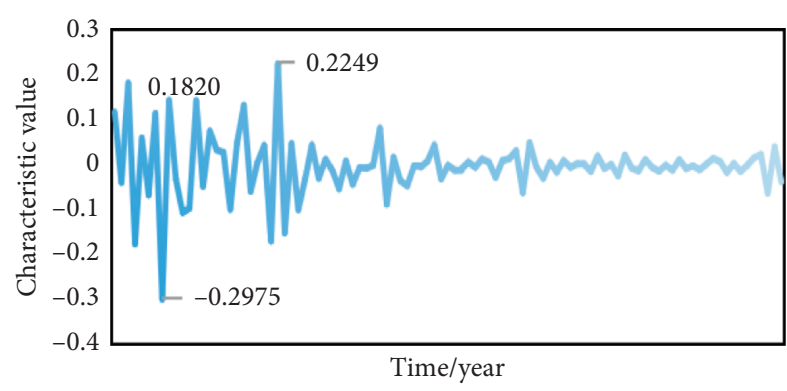

Figure 12: The changing trend of the instrumentalness.

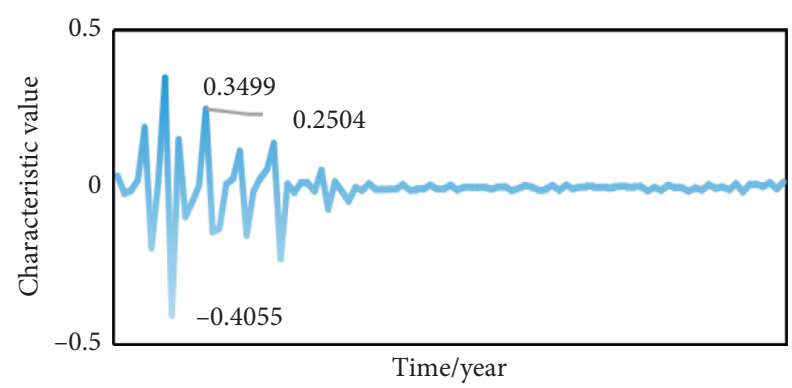

FIGURE 13: The changing trend of the speechiness.

Among them, the repertoire with revolutionary music characteristics in 1924 is not in the "influence_data" structure, so we looked for similar tracks in the past few years. So, in 1921, we found that the repertoire created by the musician ID 26350 is the one closest to revolutionary music.

Finally, according to the repertoire with the characteristics of revolutionary music, we determine the musicians who create this repertoire and calculate their music influence through the directional network of musicians' influence. The results are shown in Table 8.

\subsection{The Evolution of Music Schools}

8.3.1. The Method of Coordinate Axis Descent to Solve the Lasso Regression. Lasso is a linear model for estimating sparse coefficients. It tends to choose solutions with less nonzero coefficients, which effectively reduces the number of features on which a given solution depends. Under certain conditions, Lasso can accurately recover a set of nonzero coefficients [30].
Mathematically, it consists of a linear model and an additional regularization term. The objective function of minimization is as follows:

$$
F_{\alpha}(w)=\min _{w} \frac{1}{2 * n}\|X w-y\|_{2}^{2}+\alpha\|w\|_{1} .
$$

Therefore, lasso estimation solves the minimization of least squares penalty by adding $\alpha\|w\|_{1}$, where $\alpha$ is constant and $\|w\|_{1}$ is $L 1$ norm of the coefficient vector.

Lasso uses the coordinate axis descent method as the fitting coefficient algorithm. The coordinate axis descent method is to descend along the direction of the coordinate axis, which is different from the gradient descent. It iterates step by step through a heuristic method to find the minimum value of the function.

8.3.2. Lasso Regression. In order to analyze the influence process of a certain music genre over time, we use lasso regression to screen out the indicators that can reveal the dynamic influence factors and then study how the music genre and musicians change over time [31].

Firstly, the normalized data is used in the artist data set. The independent variable is set as the musical characteristics of the musician, and the dependent variable is set as follows:

$$
y=0.5 \times \text { prevalence }+0.5 \times \text { influence. }
$$

The results of lasso regression are as follows:

$$
\begin{aligned}
y= & -0.0356 \times \text { valence }+0.1774 \times \text { loudness } \\
& -0.1073 \times \text { acousticness. }
\end{aligned}
$$

It shows the influence of three independent variables, namely, valence, loudness, and acousticness, on the dependent variable. $y$ is more significant, which can reveal the dynamic influencers.

Secondly, we randomly select a musician and analyze the change with time according to the results of lasso regression. We selected a musician whose ID number is 26350 . Figures 14-16 show the changes of the musician's three musical characteristics: valence, loudness, and acousticness over time.

As you can see from Figures 14-16, the musician's valence with ID 26350 fluctuates between 0 and 0.8 , but in most cases, it will remain between 0.2 and 0.5 . His loudness gradually decreased with the passage of time but still remained in a typical range. His acousticness fluctuates with time, but the fluctuation intensity is very small and generally maintains a relatively stable value. Only a few times, there will be relatively large fluctuations.

Finally, we choose any genre and use the same method to analyze its changes over time. We choose the type of country music, and the three music features change with time, as shown in Figures 17-19:

From Figure 17-19, we find that the valence of the country music genre shows a downward trend over time, from 0.65 to 0.5 , which indicates that the style of country music is changing from happy to sad. The loudness of country music increases with the passage of time, but the 
TABLE 6: The discrete coefficient of musical characteristics.

\begin{tabular}{lccccc}
\hline Music characteristics & Danceability & Energy & Valence & Tempo & Loudness \\
\hline Dispersion coefficient & 0.096807841 & 0.361115449 & 0.10886604 & 0.049593269 & 0.262817345 \\
Music characteristics & Acousticness & Instrumentalness & Liveness & Speechiness & duration_ms \\
Dispersion coefficient & 0.510496275 & 0.674553721 & 0.087120516 & 0.651898231 & 0.111358323 \\
\hline
\end{tabular}

TABLE 7: Songs with revolutionary musical characteristics.

\begin{tabular}{|c|c|c|c|c|c|c|c|c|}
\hline Year & artists_id & Acousticness & $\begin{array}{l}\text { Instrumen- } \\
\text { talness }\end{array}$ & $\begin{array}{l}\text { Speech- } \\
\text { iness }\end{array}$ & $\begin{array}{l}\text { Absolute } \\
\text { distance1 }\end{array}$ & $\begin{array}{l}\text { Absolute } \\
\text { distance2 }\end{array}$ & $\begin{array}{l}\text { Absolute } \\
\text { distance3 }\end{array}$ & song_title (censored) \\
\hline 1924 & 26350 & $\leq 0.001$ & 0.681 & $\leq 0.001$ & $\leq 0.001$ & 0.097 & $\leq 0.001$ & $\begin{array}{c}\text { Symphony no. } 39 \text { in E-Flat } \\
\text { major, K. 543: IV. Finale. } \\
\text { Allegro }\end{array}$ \\
\hline 1926 & 639789 & 0.921 & $\leq 0.001$ & $\leq 0.001$ & 0.180 & $\leq 0.001$ & $\leq 0.001$ & *********** Rose \\
\hline 1927 & 403120 & 0.940 & $\leq 0.001$ & $\leq 0.001$ & 0.005 & $\leq 0.001$ & $\leq 0.001$ & Sweet Sue, Just you - Live \\
\hline \multirow[t]{2}{*}{1929} & 119107 & 0.995 & 0.967 & 0.182 & 0.403 & 0.748 & 0.303 & $\begin{array}{c}\text { Preludes: Op. }{ }^{* * *} \text { no. }{ }^{*} \text { in A- } \\
\text { Minor }\end{array}$ \\
\hline & 765773 & 0.992 & 0.734 & 0.143 & 0.400 & 0.515 & 0.342 & It's Tight like that \\
\hline 1930 & 898336 & 0.952 & $\leq 0.001$ & 0.0792 & 0.008 & $\leq 0.001$ & 0.001 & Still I'm Traveling on \\
\hline \multirow[t]{2}{*}{1935} & 591857 & $\leq 0.001$ & $\leq 0.001$ & 0.332 & $\leq 0.001$ & $\leq 0.001$ & 0.023 & $\begin{array}{l}\text { Hermit songs, Op. }{ }^{* * *} \text { church } \\
\text { bell at }{ }^{* * * * *}\end{array}$ \\
\hline & 162677 & $\leq 0.001$ & 0.493 & $\leq 0.001$ & $\leq 0.001$ & 0.002 & $\leq 0.001$ & Stay on it \\
\hline 1946 & 792507 & 0.925 & $\leq 0.001$ & $\leq 0.001$ & 0.001 & $\leq 0.001$ & $\leq 0.001$ & $\begin{array}{l}\text { ******* }^{*} \text { to Watch over Me- } \\
\text { Remastered }\end{array}$ \\
\hline
\end{tabular}

TABLE 8: The Influence of musicians with revolutionary musical characteristics.

\begin{tabular}{|c|c|c|c|c|c|}
\hline year & Var & Value & artists_id & main_genre & Power \\
\hline 1924 & Instrumentalness & 0.583954695 & 26350 & Classical & $5.31 E-44$ \\
\hline 1926 & Acousticness & 0.740919594 & 639789 & Jazz & $5.31 E-44$ \\
\hline 1927 & Acousticness & 0.934606323 & 403120 & Vocal & $7.66 E-44$ \\
\hline 1929 & Acousticness & 0.591110509 & 608701 & Blues & $7.89 E-44$ \\
\hline 1929 & Instrumentalness & 0.219461260 & 954694 & Vocal & $5.31 E-44$ \\
\hline 1929 & Speechiness & 0.485278355 & 765773 & Jazz & $5.31 E-44$ \\
\hline 1930 & Speechiness & 0.079702370 & 898336 & Blues & $5.31 E-44$ \\
\hline 1930 & Acousticness & 0.943474167 & 794401 & Pop/Rock & $5.77 E-44$ \\
\hline 1935 & Speechiness & 0.354805122 & 181694 & Vocal & $5.31 E-44$ \\
\hline 1946 & Acousticness & 0.924216926 & 792507 & Vocal & $1.47 E-43$ \\
\hline 1946 & Instrumentalness & 0.495375180 & 162677 & Jazz & $1.01 E-43$ \\
\hline
\end{tabular}

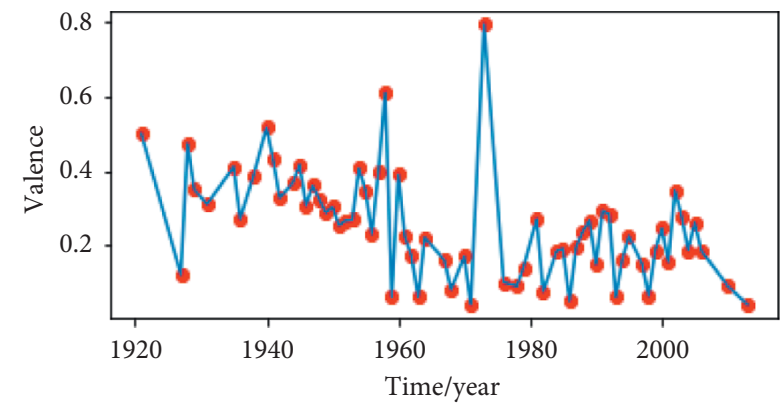

FIGURE 14: The changes of valence of the musician with ID 26350.

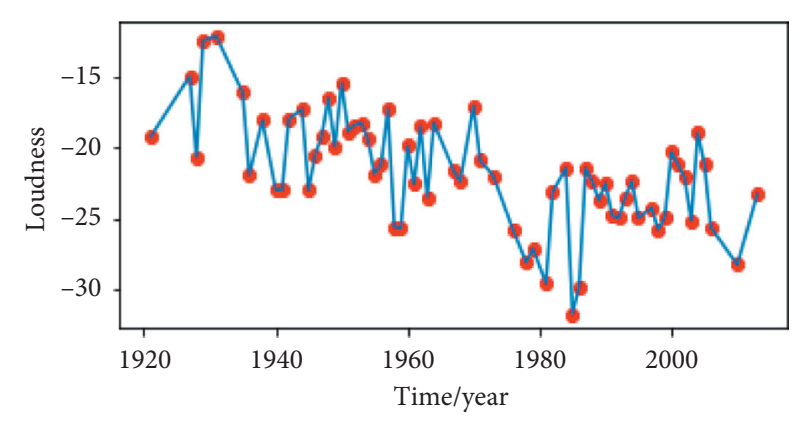

FIgURE 15: The changes of loudness of the musician with ID 26350. 


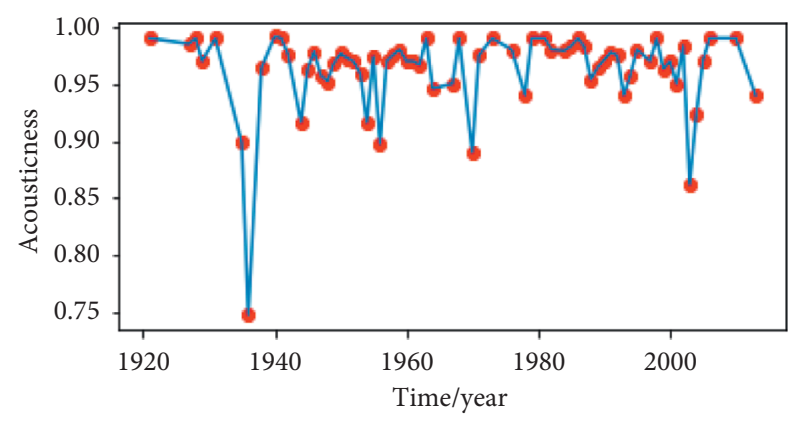

FIGURE 16: The changes of acousticness of the musician with ID 26350.

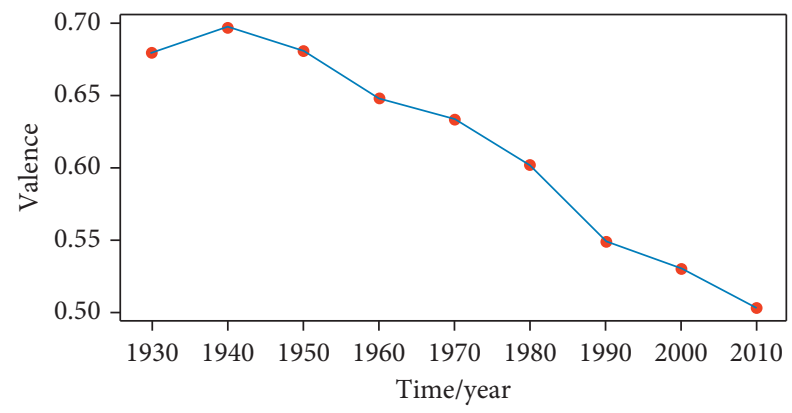

FIGURE 17: The changing trend of valence in the country genre.

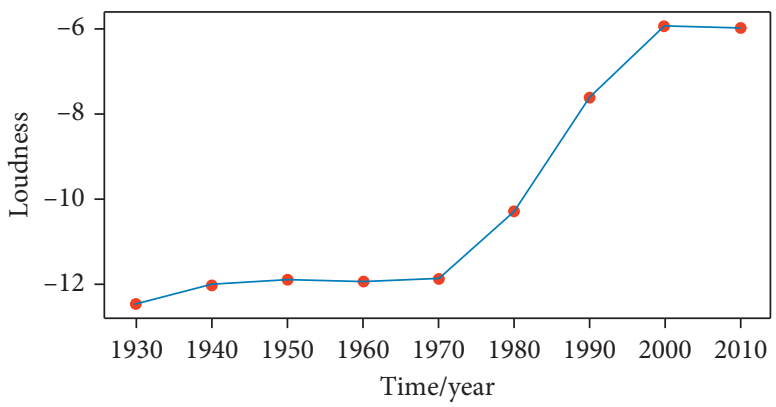

FIgURE 18: The changing trend of loudness in the country genre.

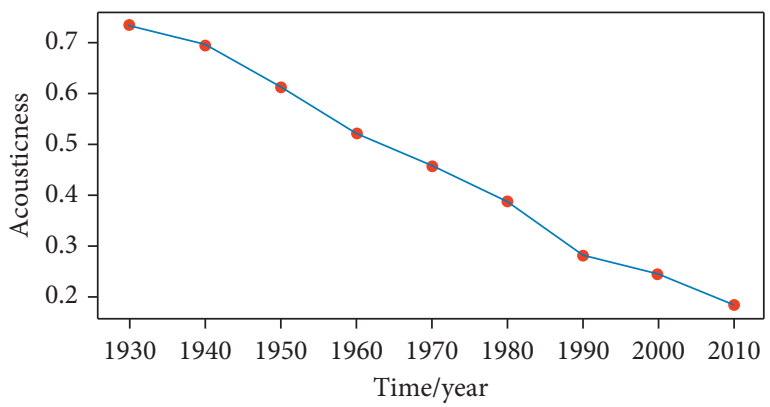

FIgURE 19: The changing trend of acousticness in the country genre.

range of change is small, and it has been maintained at about -10 . The acousticness of country music has decreased year by year, from 0.7 to 0.2 , which has changed a lot.

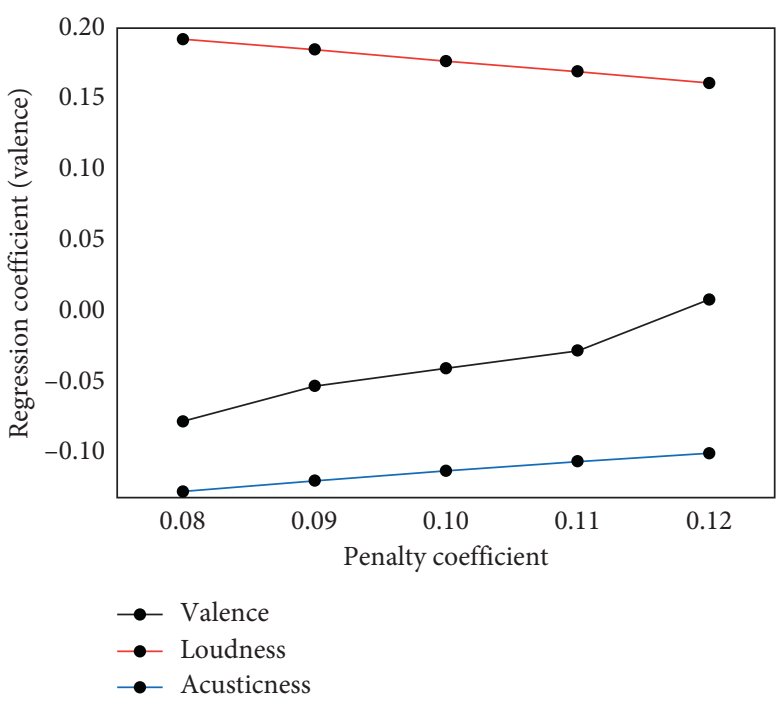

FIgURE 20: The changing of the penalty coefficient $\alpha$.

\section{Sensitivity Analysis}

In question 6, we use lasso regression to screen variables, where we set the penalty coefficient to 0.1 , and the resulting formula is as follows:

$$
\begin{aligned}
y= & -0.0356 \times \text { valence }+0.1774 \times \text { loudness } \\
& -0.1073 \times \text { acousticness. }
\end{aligned}
$$

Now, we refer to the empirical study [32], set the penalty coefficient of 0.01 interval as $0.08,0.09,0.1,0.11$, and 0.12 , and test the change of regression coefficient in different corresponding result formulas. Finally, we plot the result as a line chart, as shown in Figure 20.

It can be seen that with the increase of the penalty coefficient, the regression coefficient changes gently, and there is no violent vibration, which indicates that our model is not sensitive to the penalty coefficient and has good stability.

\section{Conclusion}

Based on PageRank, this paper establishes a dynamic analysis network of music influence by using 11 music characteristic indexes and analyzes the music influence of different genres and musicians. Using multiobjective logistic regression to establish a music similarity measurement model, this paper analyzes the music similarity between different genres and musicians combined with music influence networks to analyze whether the interaction between musicians will have a practical impact on their works. At the same time, from the perspective of music genres, using the method of two-way clustering, this paper analyzes the mutual influence and similarity between different music genres and the same music genre. Finally, the lasso region method is used to select features, explore the change factors in the process of music development, and analyze the dynamic change process of music [33-36]. 
In this paper, we choose multiobjective logistic regression, bidirectional clustering, and other methods; these methods have the characteristics of high accuracy and easy to understand. Meanwhile, this paper uses visualization tools to assist analysis many times, which helps to absorb and master information intuitively. But the analysis of this paper is also insufficient. In the process of similarity index construction, compared with direct calculation distance, the computational complexity is higher, and more computing time is needed in the face of a large amount of data. Due to the availability and quantification of data, this paper does not make full use of some discrete music features.

In the work of model improvement, we will reduce the error by increasing the number of variables and samples and analyze the deficiencies that may cause the error from the aspects of data processing, model building, and model solving. To build a model that can analyze more music features (including virtual features), we collect more information about musicians, expand the number of analysis objects, and make the model more universal in practical application.

To sum up, we use network science to build a dynamic network to analyze the similarity of music, the evolution process, and the impact of music on culture. Our research results can provide a theoretical basis for evaluating the influence of different music schools and have certain research significance and practical value in the fields of music, history, social science, and practice.

\section{Data Availability}

The data used to support the findings of this study are included within the article.

\section{Conflicts of Interest}

The authors declare that there are no conflicts of interest regarding the publication of this paper.

\section{Acknowledgments}

This study was funded by the General Project of Philosophy and Social Science Planning of Anhui Province: Research on Government R\&D Subsidies Promoting Green Innovation Efficiency of Anhui High-Tech Industries (no: AHSKY2019D085), the Teaching and Research Fund Project of the Education Department of Anhui Province (2020jyxm0017), "First-Class Course" of Anhui University of Finance and Economics (acylkc202008), and the Teaching and Research Fund Project of the Anhui University of Finance and Economics (acjyyb2020011 and acjyyb2020014).

\section{References}

[1] D. Naser and G. Saha, "Influence of music liking on EEG based emotion recognition," Biomedical Signal Processing and Control, vol. 64, 2021.

[2] A. Banerjee, S. Shankha, S. Roy et al., "A novel study on perception-cognition scenario in music using deterministic and non-deterministic approach," Physica A: Statistical Mechanics and Its Applications, vol. 567, 2021.

[3] Y. Zhuang and F. Q. Yu, "Music genre classification based on modulation spectrum features of rhythmand rhyme," Computer Engineering, vol. 41, no. 1, pp. 186-189, 2015.

[4] G. Suganda, W. Antoni, and Roslynlia, "Neural collaborative for music recommendation system," in Proceedings of the IOP Conference Series: Materials Science and Engineering, Suzhou, China, March 2021.

[5] J. X. Li, L. X. Han, X. S. Li et al., "An Evaluation of Deep Neural Network Models for Music Classification Using Spectrograms," Multimedia Tools and Applications, 2021.

[6] J. Dai, S. Liang, W. Xue et al., "Long short-term memory recurrent neural network based segment features for music genre classification," in Proceedings of the International Symposium on Chinese Spoken Language Processing (ISCSLP), pp. 1-5, Tianjin, China, October 2016.

[7] J. Sim, T. Garry, and J. Jessica, "Understanding and predicting the motivators of mobile music acceptance-a multi-stage MRA-artificial neural network approach," Telematics and Informatics, vol. 31, 2014.

[8] H. Yuan, S. S. Tseng, G. Wu et al., "A two-phase feature selection methods using both filter and wrapper," in Proceedings of the 1999 IEEE International Conference on Systems, Man, and Cybernetics, no. 2, pp. 132-136, Tokyo, Japan, October 1999.

[9] R. Setiono and H. Liu, "Neural-network feature selector," IEEE Transactions on Neural Networks, vol. 8, no. 3, pp. 654-662, 1997.

[10] H. M. Lee, C. M. Chen, J. M. Chen, and Y. L. Jou, "An efficient fuzzy classifier with feature selection based on fuzzy entropy," IEEE Transactions on Systems, Man, and Cybernetics. Part B, Cybernetics: A Publication of the IEEE Systems, Man, and Cybernetics Society, vol. 31, no. 3, pp. 426-432, 2001.

[11] E. Wang, "A new smooth method based on rotated hyperbola for support vector machine in classification," Journal of Physics: Conference Series, vol. 1074, 2018.

[12] J. J. Burred and A. Lerch, "A hierarchical approach to automatic musical genre classification," in Proceedings of the Conference on Digital Audio Effects, London, UK, September 2003.

[13] Y. H. Cheng, C. C. Pang, M. N. Yang, and K. N. Chen, "Automatic music genre classification based on CRNN," Engineering Letters, vol. 29, 2020.

[14] S. Nilson, R. F. Gabriel, and R. Fujita, "Preferred music genre benefits during strength tests: increased maximal strength and strength-endurance and reduced perceived exertion," Perceptual and Motor Skills, vol. 128, 2020.

[15] X. Y. Yang, Y. Z. Dong, and J. Li, "Review of data featuresbased music emotion recognition methods," Multimedia Systems, vol. 24, 2017.

[16] C. Zhen, S. Song, and J. P. Xu, "Study on multi-modal music genre classification," Journal of Fron-Tiers of Computer Science and Technology, vol. 5, no. 1, pp. 50-58, 2011.

[17] M. A. Ali and Z. A. Siddiqui, "Automatic music genres classification using machine learning," International Journal of Advanced Computer Science and Applications (IJACSA), vol. 8, no. 8, pp. 337-344, 2017.

[18] G. Tzanetakis and P. Cook, "Musical genre classification of audio signals," IEEE Transactions on Speech and Audio Processing, vol. 10, no. 5, pp. 293-302, 2002.

[19] C. Zopluoglu, "Detecting examinees with item preknowledge in large-scale testing using extreme gradient boosting 
(XGBoost)," Educational and Psychological Measurement, vol. 79, no. 5, pp. 931-961, 2019.

[20] D. Goldfarb, S. Ma, and K. Scheinberg, "Fast alternating linearization methods for minimizing the sum of two convex functions," Mathematical Programming, vol. 141, no. 1-2, pp. 349-382, 2013.

[21] T. Leandro, F. Vicent Jose, and Y. Gevorg, "An algorithm for ranking the nodes of multiplex networks with data based on the PageRank concept," Applied Mathematics and Computation, vol. 392, 2021.

[22] Z. L. Tian, Y. Zhang, J. X. Wang, and C. Gu, "Several relaxed iteration methods for computing PageRank," Computational and Applied Mathematics, vol. 388, 2021.

[23] H. Ling, PageRank and K in Web Data Mining_ Research on the Improvement of Means Algorithm, Lanzhou Jiaotong University, Lanzhou, China, 2019.

[24] F. Prataviera, M. Edwin, M. Ortega, and M. Gauss, "A new generalized odd log-logistic flexible Weibull regression model with applications in repairable systems," Reliability Engineering \& System Safety, vol. 178, 2018.

[25] S. X. Cheng, H. Zhan, H. Q. Yao et al., "Large-scale manyobjective particle swarm optimizer with fast convergence based on Alpha-stable mutation and Logistic function," Applied Soft Computing, vol. 99, 2021.

[26] D. Vu and A. Murray, "Variational algorithms for biclustering models," Computational Statistics \& Data Analysis, vol. 89, no. 1, pp. 12-24, 2015.

[27] C. Chi Eric and K. Lange, "Splitting methods for convex clustering," Journal of Computational and Graphical Statistics, vol. 24, 2015.

[28] S. Nieminen, E. Istók, E. Brattico et al., "The development of aesthetic responses to music and their underlying neural and psychological mechanisms," Cortex, vol. 47, 2011.

[29] Anonymous, "Music revolution: the middle east and Africa forum," Materials Performance, vol. 58, no. 7, 2019.

[30] Y. Y. Shi, J. Huang, Y. L. Jiao et al., "Generalized Newton-Raphson algorithm for high dimensional LASSO regression," Statistics and Its Interface, vol. 14, 2021.

[31] M. Yazdi, N. A. Golilarz, A. Nedjati et al., "An improved lasso regression model for evaluating the efficiency of intervention actions in a system reliability analysis," Neural Computing and Applications, pp. 1-16, 2021.

[32] M. B. Mathur and T. J. VanderWeele, "Sensitivity analysis for unmeasured confounding in meta-analyses," Journal of the American Statistical Association, vol. 115, no. 529, pp. 163172, 2020.

[33] J.-M. Zhu, L. Wang, and J.-B. Liu, "Eradication of Ebola based on dynamic programming," Computational and Mathematical Methods in Medicine, vol. 2016, Article ID 1580917, 9 pages, 2016.

[34] J. B. Liu, J. Zhao, and J. Min, "On the Hosoya index of graphs formed by a fractal graph," Fractals-Complex Geometry Patterns and Scaling in Nature and Society, vol. 27, no. 8, pp. 19-35, 2019.

[35] J.-M. Zhu, Y. Chen, and S. Zhang, "Analysis of the impact of climate change on national vulnerability based on fuzzy comprehensive evaluation," Discrete Dynamics in Nature and Society, vol. 2020, Article ID 3527540, 10 pages, 2020.

[36] F. Xu, Y.-A. Du, H. Chen et al., "Prediction of fish migration caused by ocean warming based on SARIMA model," Complexity, vol. 2021, Article ID 5553935, 9 pages, 2021. 